\title{
Bilateral Lateral Rectus Palsy in Children with Scrub Typhus
}

\author{
Sudeep K. $C^{1} \cdot$ Muthuvel $R^{1} \cdot$ Harshita Nori ${ }^{1}$ Prithviraj $K^{1} \cdot$ Karthi Nallasamy $^{1}$ - Suresh Kumar Angurana ${ }^{1}$
}

Received: 30 October 2021 / Accepted: 24 November 2021 / Published online: 22 January 2022

(c) Dr. K C Chaudhuri Foundation 2022

To the Editor: Central nervous system (CNS) manifestations in scrub typhus are reported in 7.4\%-29\% children including meningoencephalitis, aseptic meningitis, cerebellitis, cranial nerve palsies, transverse myelitis, Guillan-Barré syndrome, and brachial neuritis [1]. Occurrence of isolated sixth cranial nerve palsy with scrub typhus is rare and limited to few case reports [2-4]. We present two children of scrub typhus with isolated bilateral lateral rectus palsy, which resolved with treatment.

Case 1 was a 6-y-old female who presented with fever for $5 \mathrm{~d}$ followed by respiratory distress, lethargy, and diplopia for $1 \mathrm{~d}$. Examination revealed mild pallor, periorbital edema, eschar, respiratory distress, hepatosplenomegaly, and Glasgow Coma Scale (GCS) of 13/15. Case 2 was a 9-y-old male who presented with fever for $7 \mathrm{~d}$ followed by headache and diplopia for $2 \mathrm{~d}$. Examination revealed pallor, periorbital edema, rash, eschar, generalized lymphadenopathy, and hepatosplenomegaly. Both the cases had medial deviation of both the eyes with no lateral movement.

Investigations revealed leucocytosis, thrombocytopenia, hyponatremia, mild transaminitis, normal brain imaging (CT in case 1 and MRI in case 2), normal cerebrospinal fluid (CSF) analysis (in case 1), sterile blood and CSF cultures, positive scrub typhus ELISA, and negative workup for dengue, malaria, enteric fever. Treatment included intravenous doxycyline (for $7 \mathrm{~d}$ ) and ceftriaxone (stopped after confirmed diagnosis), which led to defervesence by $48-72 \mathrm{~h}$, improvement in eye movements by day $4-5$, and discharge after a hospital stay of 5-6 d. Both were doing fine at 2-wk follow-up with normal eye movements and vision.
CNS manifestations of scrub typhus are possibly due to vasculitis of cerebral cortex and cranial nerve nuclei; and leucocytoclastic vasculitis of vasa nervosum of cranial nerves leading to meningoencephalitis and cranial nerve palsy [1]. Although vasculitis is main pathology in scrub typhus, the treatment remains doxycycline with no role of steroids or immunotherapy, as in index report.

\section{Declarations}

Conflict of Interest None.

\section{References}

1. Nallasamy K, Gupta S, Bansal A, et al. Clinical profile and predictors of intensive care unit admission in pediatric scrub typhus: a retrospective observational study from North India. Indian J Crit Care Med. 2020;24:445-50.

2. Ozair A, Kumar V, Himanshu D, Bhat V. A rare etiology behind isolated lateral rectus palsy: scrub typhus. J Family Med Prim Care. 2020;9:2538-40.

3. Ete T, Mishra J, Barman B, Mondal S, Sivam RK. Scrub typhus presenting with bilateral lateral rectus palsy in a female. J Clin Diagn Res. 2016;10:OD16-7.

4. Chauhan A, Jandial A, Mishra K, Sandal R. Scrub typhus and lateral rectus palsy: an uncommon presentation of a common illness. BMJ Case Rep. 2021;14:e240882.

Publisher's Note Springer Nature remains neutral with regard to jurisdictional claims in published maps and institutional affiliations.

Suresh Kumar Angurana sureshangurana@gmail.com

1 Division of Pediatric Critical Care, Department of Pediatrics, Advanced Pediatrics Center, Postgraduate Institute of Medical Education and Research (PGIMER), Chandigarh 160012, India 